\title{
Measures of Rheumatoid Arthritis Disease Activity in Australian Clinical Practice
}

\author{
Andrew Taylor ${ }^{1}$ and Hanish Bagga ${ }^{2}$ \\ ${ }^{1}$ St. John of God Medical Centre, Suite 19/100 Murdoch Drive, Murdoch, WA 6150, Australia \\ ${ }^{2}$ Mid North Coast Arthritis Clinic, Suite 1, 18-20 Scarba St., Coffs Harbour, NSW 2450, Australia \\ Correspondence should be addressed to Andrew Taylor, altaylor@iinet.net.au
}

Received 13 January 2011; Accepted 1 March 2011

Academic Editor: M. Harth

Copyright (C) 2011 A. Taylor and H. Bagga. This is an open access article distributed under the Creative Commons Attribution License, which permits unrestricted use, distribution, and reproduction in any medium, provided the original work is properly cited.

Objectives. To investigate which rheumatoid arthritis (RA) disease activity measures are being collected in patients receiving glucocorticoids, non-biologic or biologic disease-modifying antirheumatic drugs (DMARDs) in Australian rheumatology practice. Methods. A retrospective audit of medical records was conducted from eight rheumatology practices around Australia. Each rheumatologist recruited 30 consecutive eligible patients into the review, 10 of whom must have been receiving a biological agent for rheumatoid arthritis. Disease activity measures and radiographic assessments were collected from each patient's last consultation. For biologic patients, disease activity measures were also collected from when the patient was first initiated on the biological agent. Results. At last consultation, the disease measures that were recorded most often were ESR (89.2\%), haemoglobin $(87.5 \%)$, and CRP $(84.2 \%)$. DAS28 was infrequently recorded $(16.3 \%)$. The rate of recording disease activity measures for patients receiving biologic DMARDs decreased over time (mean 27 months). Conclusion. This review has shown inconsistency of RA activity measures being recorded in Australian rheumatology clinical practice. An accurate assessment of the disease process is necessary to effectively target rheumatoid arthritis patients to treat in order to achieve optimal outcomes.

\section{Introduction}

Rheumatoid arthritis (RA) is a chronic systemic inflammatory autoimmune disease that has a highly variable presentation and time course. The symptoms and signs of RA may vary from joint complaints, such as pain, stiffness, swelling to functional impairment, fatigue, and loss of general health [1]. Importantly, persistently high disease activity can lead to irreversible joint damage [2], disability [3], and even a reduction in life expectancy [4]. Quantitative assessment of RA differs from many other clinical conditions in that a single "gold standard" measure, which can be used to assess all patients, is not available for the diagnosis, prognosis, or monitoring of RA.

Disease activity measures are useful in RA as they reflect patient outcome and response to therapy in clinical care and in clinical trials. Various measures exist to provide information about the various dimensions of outcomes, for example, pain, disability, and impairment. However, due to differences in disease expression of RA, many disease measures have limitations, for example, joint counts have poor reproducibility; the erythrocyte sedimentation rate (ESR) and C-reactive protein (CRP) are poor diagnostic measures as they are normal in $40 \%$ of patients with RA; radiographs change slowly, reflecting cumulative damage rather than acting as an early-stage diagnostic test [5].

In order to standardise the assessment of RA in clinical trials of antirheumatic agents, in 1993, the American College of Rheumatology (ACR) recommended core sets of disease activity variables to be measured $[6,7]$. These include 7 core measures: swollen joint count, tender joint count, patient assessment of global status, an acute phase reactant (ESR or CRP), and health professional assessment of global status, physical function, and pain. However, use of the ACR criteria is limited by the fact that they provide relative measure of response and cannot be used to describe a patient's disease activity at a specific point in time or to compare disease activity states between individual patients or cohorts of 
patients [8]. For these reasons, indices that allow continuous measures of disease activity have also been developed.

In 2008, the European League Against Rheumatism (EULAR), together with the ACR, developed guidelines for response criteria based on the Disease Activity Score (DAS) and the modified DAS score-DAS28 [9]. These scores, which are composed of four measures (tender joint count, swollen joint count, acute phase reactant laboratory test, and patient estimate of global status), allow changes in RA status to be determined and have recently been validated for use in RA clinical trials [10]. These disease measures and ACR response criteria have shown comparable performance and validity in RA studies [9-11].

With the arrival of new therapeutic options in RA, such as biological agents, remission is now considered to be the ultimate goal of treatment. Defining treatment targets can help facilitate treatment changes to occur within distinct timeframes according to defined algorithms $[12,13]$. This concept of "treating to target" has been widely adopted in practice for many diseases including diabetes, hyperlipidaemia, and hypertension. Treating RA strategically according to defined outcome targets leads to significantly better clinical outcomes than traditional means of followup [12]. Recently, an international task force (T2T) recommended regular follow-up every 1 to 3 months during active disease with appropriate therapeutic adaptation to reach either remission in early disease or low disease activity in long-standing disease within 3 to 6 months [14]. It is recommended that a validated composite measure of disease activity, which includes frequent joint assessment and imaging should be used in routine clinical practice to guide treatment decisions [13].

Due to the difficulty in applying the ACR criteria for remission in clinical trials [8], many trials have used the DASbased definitions. In a recent randomised trial, both DAS and DAS28 remission results were found to be comparable for assessing, achieving, and sustaining remission in RA. Therefore, it is important for physicians to choose the correct outcome measures and indices that can be used in clinical practice to assess treatment effectiveness and remission. In the clinical practice setting, however, little attention and guidance have been given about what to monitor, which assessments to use, and how to define response and remission of RA [1]. The Australian Rheumatology Association recommends using DAS28 to monitor patients receiving a biological agent in clinical practice [12]. In Australia, the Pharmaceutical Benefits Scheme (PBS) requires an assessment of treatment response based on ESR, CRP, or active joint count for the prescription of reimbursable biological disease-modifying antirheumatic drugs (bDMARD) [15]. There may be little time during consultations to perform a detailed evaluation, such as the ACR core criteria, for every patient. Consequently, standard rheumatology care may be delivered largely based on qualitative impressions rather than quantitative data collection [5], leading to disparities in the assessment of RA activity between rheumatology practices.

The aim of this Australian quality assurance review was to investigate which RA disease measures are being collected in conventional rheumatology practice and what differences may exist in these measures for patients receiving biological therapy, compared with patients receiving steroids or DMARDs. This was conducted via a retrospective audit of patient medical records.

\section{Methods}

2.1. Rheumatology Practices and Patients. A total of eight rheumatologists, from New South Wales, Queensland, Victoria, and Western Australia participated in this observational, nonrandomised, pilot quality assurance review between December 2008 and February 2009. The patient population reviewed represented a cross-sectional cohort of patients in predominantly private practice. Each rheumatologist recruited 30 consecutive eligible patients into the review by retrospectively reviewing the medical records of those patients who visited their practice for consultation as of 1 April 2008. Ten of the 30 patients must have been receiving a biological agent for RA (classified as "biologic patients") at the time of the audit while the remaining 20 patients must have been receiving a nonbiological agent for RA (e.g., steroids and/or DMARD; classified as "nonbiologic patients"), with no previous history of biological use. Patient privacy was maintained throughout the review using nonidentifiable patient ID numbers. The review was conducted in accordance with the recommendations of the Australian National Health and Medical Research Council (NHMRC) [14]. The NHMRC states that a quality assurance review can proceed without review by a Human Research Ethics Committee as long as patient consent has been obtained, privacy is maintained, and patients will not suffer any burden or harm.

2.2. Data Collection. Data were collated and analysed by the independent audit administrator, Discovery Sydney, Walsh Bay, NSW. The primary objectives of the audit were to assess which RA disease measurements are being collected in routine clinical practice and how frequently these measures are taken via retrospective analyses of patient data records. The secondary objective was to investigate whether data collection varies between those patients treated with DMARDs and bDMARDs.

Rheumatologists documented each patient's demographics and medical history by referring to the patient's medical records. This included age, sex, and the type and number of treatments, including steroids and DMARDs. The following disease activity measures were recorded from each patient's last consultation: swollen joint count, tender joint count, CRP, ESR, morning stiffness, haemoglobin, rheumatoid factor (RF), disease activity score 28 (DAS28), physician's and patient's global assessment of disease activity, Health Assessment Questionnaire-Disability Index (HAQ-DI), and any other standard measures that may have been used by the rheumatologist (e.g., Simplified Disease Activity Index). Radiographic assessments were also audited. For biologic patients, in addition to recording these disease measures at their last consultation, they were also recorded from when the patient was first initiated on the biological agent. 
Table 1: Patient demographics and treatments.

\begin{tabular}{lccc}
\hline & $\begin{array}{c}\text { All patients } \\
(n=240)\end{array}$ & $\begin{array}{c}\text { Biologic patients } \\
(n=80)\end{array}$ & $\begin{array}{c}\text { Nonbiologic patients } \\
(n=160)\end{array}$ \\
\hline Mean age, years (range) & $58.6(15-85)$ & $55.4(15-82)$ & $60.2(23-85)$ \\
Female (\%) & 70 & 75 & 68 \\
Receiving a steroid at last consultation (\%) & 51.7 & 51.3 & 51.9 \\
Receiving a DMARD at last consultation (\%) & 92.1 & 83.8 & 96.3 \\
Number of DMARDs prescribed (\%) & & & 3.8 \\
$\quad$ Zero & 7.9 & 16.3 & 51.3 \\
One & 56.3 & 66.3 & 36.3 \\
Two & 29.2 & 15.0 & 8.8 \\
Three & 6.7 & 2.5 & 81.3 \\
Type of DMARD (\%) & & & 37.5 \\
Methotrexate & 79.2 & 75.0 & 16.3 \\
Leflunomide & 29.6 & 13.8 & 11.3 \\
Hydroxychloroquine & 13.8 & 8.8 & 3.8 \\
Sulfasalazine & 9.2 & 5.0 & \\
Other (azathioprine, 6mercaptopurine, cyclosporine) & 2.9 & 1.3 & \\
\hline
\end{tabular}

2.3. Statistical Analyses. Results for disease measure recording were reported as percentages. Statistical analysis on the frequency of recording a particular RA disease activity measure (including radiographic data) between biologic and nonbiologic patients was done using a Chi-squared test with one degree of freedom. A Mc Nemar's test with one degree of freedom was used to evaluate any significant difference between the frequencies of recording a particular RA activity measure at the initiation of a biologic compared with the patient's last consultation. Mean \pm standard errors (SE) were calculated for drug doses, and a Kruskal-Wallis rank sum test with one degree of freedom was used to evaluate any significant difference between doses prescribed to biologic and nonbiologic patients. A $P$-value $<.05$ was considered significant.

\section{Results}

3.1. Patient Demographics and Treatments. The patient demographics and current treatments of all enrolled patients $(n=240)$ are shown in Table 1 . The mean patient age was 58.6 years (standard deviation 13.4; range 15-85 years), and reflective of the general RA population in that a higher proportion of patients enrolled were female (70\%). In terms of treatments, more biologic patients received a steroid (prednisolone) at some point in their management plan for RA compared with nonbiologic patients $(90.0 \%$ versus $80.0 \%$, resp.). However, the percentage of patients receiving prednisolone at their last consultation was similar between the two groups $(51.3 \%$ versus $51.9 \%$, resp.). The mean prednisolone dose for all patients was $5.77 \pm 0.27 \mathrm{mg}$ /day; there was no significant difference in dose between biologic and nonbiologic patients $(6.35 \pm 0.5 \mathrm{mg} /$ day versus $5.72 \pm$ $0.31 \mathrm{mg} /$ day; $P=.9054$ ). A high proportion of biologic and nonbiologic patients were receiving treatment with a DMARD $(83.8 \%$ and $96.3 \%$, resp.) at their last consultation. Over half of all patients were receiving one DMARD, the most common being methotrexate $(79.2 \%$; mean dose $16.2 \pm 0.41 \mathrm{mg} /$ day). As per protocol, all biologic patients were receiving a biologic treatment at their last consultation and no nonbiologic patient had previously received a biological agent.

3.2. Disease Measures. A summary of the RA measures reported in all patients, nonbiologic patients, and biologic patients is shown in Figure 1. At the patients' last consultation, the disease measures that were recorded most often for the overall patient population were ESR (89.2\%), haemoglobin $(87.5 \%)$, and CRP $(84.2 \%)$. Swollen joint counts and tender joint counts were documented in the medical records of approximately half of all patients enrolled (53.8\% for both measures) while RF was reported for approximately a third of all patients (30.8\%). DAS28 $(16.3 \%)$, morning stiffness (13.3\%), and the patient's global assessment of disease activity $(11.3 \%)$ were recorded infrequently. The physician's global assessment of disease activity was very rarely measured $(0.4 \%)$, and HAQDI was never taken. No other RA activity measures or indices were documented.

3.3. Biologic versus Nonbiologic Patients. Among nonbiologic patients, the only measures that were documented in more than $90 \%$ of patients were haemoglobin $(95.6 \%)$ and ESR (94.4\%). In comparison, the documentation of these two measures for biologic patients occurred significantly less frequently: $71.3 \%(P<.0001)$ and $78.8 \%(P<.001)$, respectively. Swollen and tender joint counts were occasionally recorded in nonbiologic patients ( $48.8 \%$ for both measures) while in biologic patients these measures were recorded significantly more often $(63.8 \%$ for both measures; $P<.05)$. 


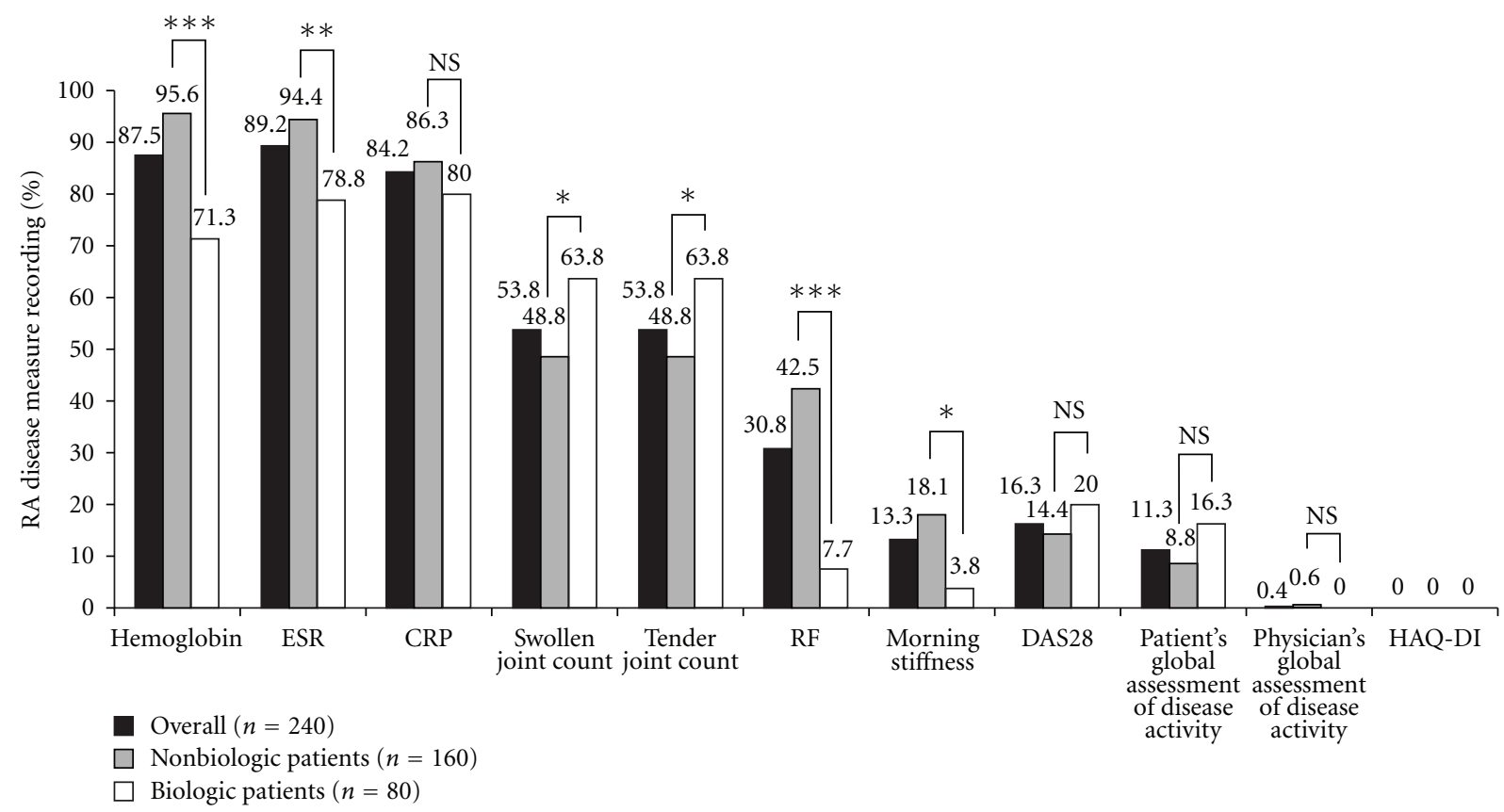

FIGURE 1: Frequency of RA disease measure recording for all patients, nonbiologic patients, and biologic patients at their last consultation. Statistical significance between nonbiologic and biologic patients (Chi-squared test, 1 degree of freedom): ${ }^{* * *} P<.0001 ;{ }^{* *} P<.001$; ${ }^{*} P<.05 ;$ NS: not significant.

RF was measured in approximately half of all nonbiologic patients $(42.5 \%)$ while in biologic patients it was documented significantly less often $(7.5 \% ; P<.0001)$. Morning stiffness was documented significantly more often for nonbiologic patients than biologic patients $(18.1 \%$ versus $3.8 \% ; P<.05)$. No significant difference was seen between nonbiologic and biologic patients in the recording of CRP, DAS28, patient's global assessment of disease activity, physician's assessment of disease activity, or HAQ-DI.

3.4. Biologic at Initiation versus Last Visit. The mean period of time from a patient being initiated on a biologic to last consultation was 27 months. The change in RA disease measure recording between initiation of a biologic and last consultation is shown in Figure 2. At the initiation of a biologic, the five disease measures of swollen joint count $(92.5 \%)$, tender joint count $(90.0 \%)$, ESR $(88.8 \%)$, CRP $(88.8 \%)$, and haemoglobin $(82.5 \%)$ were frequently recorded. RF was occasionally taken at initiation $(45 \%$ of patients) while DAS28, patient's global assessment of disease activity, morning stiffness, and HAQ-DI score were all recorded infrequently $(\leq 22.5 \%)$. The physician's global assessment of disease activity was never taken. In general, the frequency that these measurements were recorded decreased over time. At the patients' last consultation, only CRP was regularly assessed at consultation $(80 \%$ of patients). Measures of swollen joint count and tender joint count were both significantly reduced to an incidence of 63.8\% $(P<.0001)$, compared with approximately 90\% when the patient's biologic was initiated. The documentation of haemoglobin was also significantly reduced $(82.5 \%$ versus $71.3 \% ; P<.05)$ while $\mathrm{RF}$ showed the largest significant drop in recording with a decrease from $45 \%$ at initiation to $7.5 \%$ at last consultation $(P<.0001)$. ESR, CRP, patient's global assessment of disease activity, DAS28, morning stiffness, and HAQ-DI were all reduced compared with initiation, although these reductions were not statistically significant. The physician's global assessment of disease activity was never taken at the patient's last visit.

3.5. Radiographic Data. The proportion of patients with radiographic data available in their medical records was similar between biologic (70.0\%) and nonbiologic patients (76.9\%). The most common radiographic method for both groups was the use of X-ray (91.1\% biologic, $89.4 \%$ nonbiologic; $P=$ NS). CT scans and ultrasound images were used rarely for both the biologic and nonbiologic groups (CT scan: $8.9 \%$ biologic versus $8.1 \%$ nonbiologic; ultrasound: $7.1 \%$ biologic versus $8.1 \%$ nonbiologic; both comparators $P=$ NS). MRI data were taken more often for biologic patients than nonbiologic patients $(23.2 \%$ versus $17.1 \%$, resp.), although this difference was not significant. Of those patients with radiographic data in their records, approximately one in five nonbiologic patients had evidence of erosive disease $(22.8 \%)$ and/or disease progression (17.9\%). Significantly more biologic patients had documented evidence of erosive disease $(41.1 \% ; P<.05)$ compared with nonbiologic patients $(22.8 \%)$. Although the documentation of disease progression was also higher in biologic patients (30.4\%) compared with nonbiologic patients (17.9\%), this finding was not significant. 


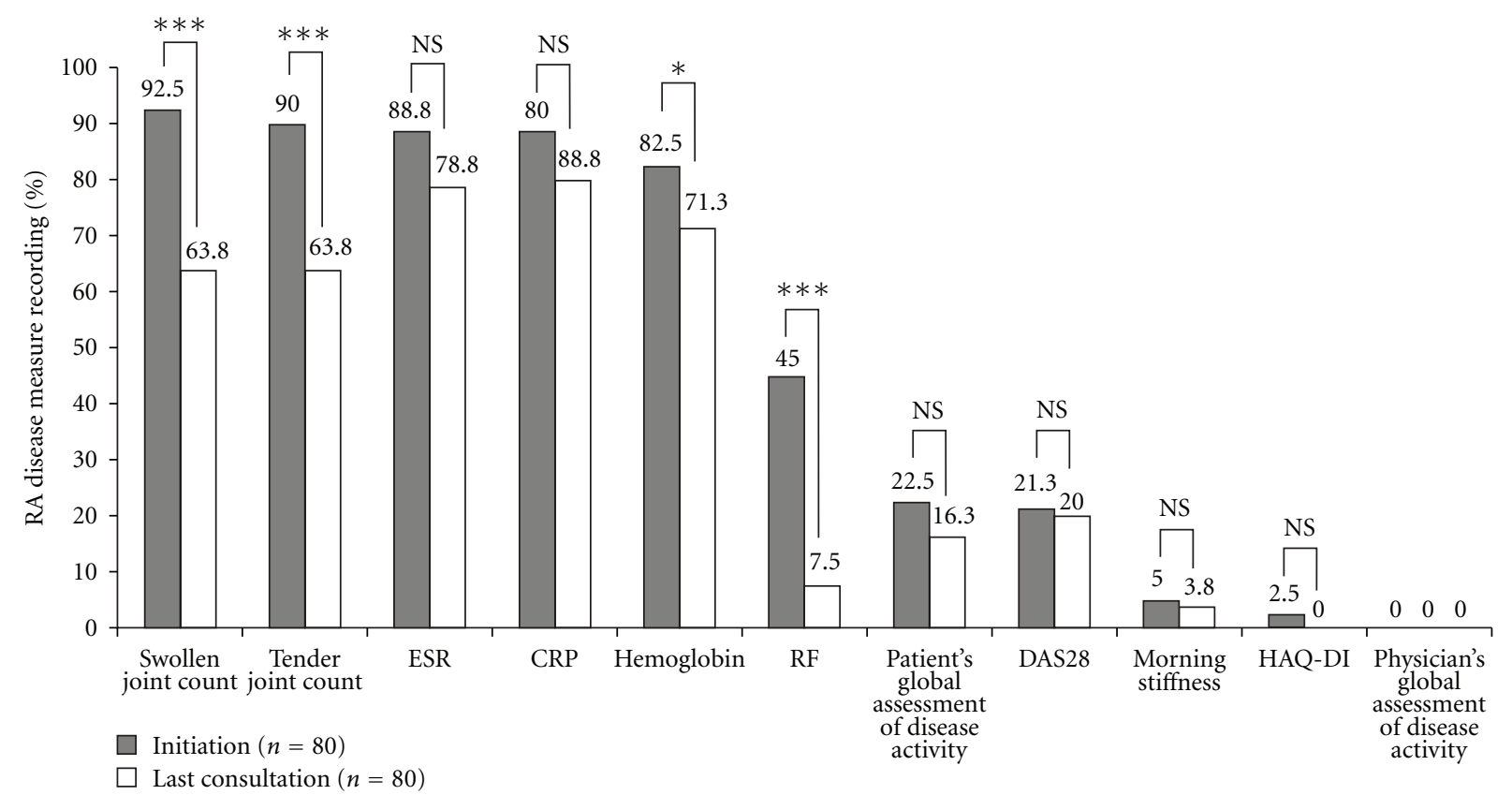

Figure 2: Frequency of RA disease measure recording for biologic patients at the initiation of a biologic and at their last consultation. Statistical significance between nonbiologic and biologic patients (McNemar's test, 1 degree of freedom): ${ }^{* * *} P<.0001$; ${ }^{*} P<.05$; NS: not significant.

\section{Discussion}

It has been traditional in clinical practice to monitor disease status as well as the efficacy and toxicity from therapy with individual outcome measures in RA. Guidelines exist that recommend outcome measures for clinical trials in RA, but, until recently, there were no formal guidelines to advise rheumatologists on which disease activity measures should be regularly monitored in daily clinical practice. Certain measures are only required to be recorded in order to fulfil the PBS requirements for bDMARD initiation and continuation. However individual measures do not fully reflect the complex reality of RA disease activity, hence the development of composite indices, such as the ACR criteria, DAS, and DAS28 [16]. The Australian Rheumatology Association encourages rheumatologists to record DAS28 at each visit [14].

The aim of this observational, nonrandomised, quality assurance review was to gain knowledge on which measures are being collected in Australian rheumatology practice by retrospectively reviewing the medical records of patients with RA. The results of this review show that RA disease activity measures are not only used infrequently but that there is a lack of consistency with which RA disease measures are recorded in rheumatology clinical practice within Australia. The low recording of RA disease measures observed in this quality assurance review has been mirrored in other studies within Australia and the rest of the world, which have shown that many of the measures used in clinical trials are infrequently used in clinical practice [17-20]. In addition, to our knowledge, this is the first review to show that differences exist in the number and types of RA disease measures that are captured for patients receiving biological treatments, compared with steroids or DMARDS, and that this rate of recording disease measures for biologic patients decreases over time, from when a patient was initiated on a biological agent to their last consultation.

Several trials, the Tight Control for Rheumatoid Arthritis (TICORA), BeSt, and the Computer-Assisted Management for Early Rheumatoid Arthritis (CAMERA) studies [21-23], have shown the beneficial effect of a tight control strategy in the treatment of RA. Notably, the TICORA study has shown that a strategy of intensive outpatient management of RA, using DAS28 scores as a guide, can significantly improve disease activity. Radiographic disease progression, physical function, and quality of life were also shown to be improved [22]. In addition, the use of this validated composite score may enhance communication and uniformity between health professionals and the quality of care provided [24]. An international task force has published guidelines recommending treatment to target by measuring disease activity and adjusting therapy accordingly to optimise outcomes in rheumatoid arthritis [13]. It is recommended that validated composite measures of disease activity, which include joint assessments, be obtained and documented regularly in routine clinical practice to guide treatment decisions. However, in this current review, the most commonly used composite measure of disease activity, the DAS28, was rarely recorded for either biologic or nonbiologic patients.

Although these findings are important, the limitations of this review must be noted. Firstly, the data are from a small subset of rheumatologists who were not chosen at random, 
but who were invited to participate. As a consequence, the patient population reviewed represented a cross-sectional cohort of patients in predominantly private practice. Secondly, since the review was nonrandomised and observational in nature, it relied on rheumatologists returning data in a nonbiased and accurate manner. Finally, the review assumed that rheumatologists conduct a set of RA activity measurements at every patient consultation, which may not always be the case.

Nevertheless, our findings do raise a number of questions about the management of patients with RA in Australian rheumatology practice. Notably, are patients having their disease monitored appropriately in order to assess disease activity, remission, and progression, and consequently, are optimal treatments being chosen to control their disease? A number of reasons as to why these outcome measures are not being measured and recorded regularly at each consultation may exist. Possible barriers to regular monitoring may include lack of time and support staff during consultations, unfamiliarity with scores and difficulty with their interpretation, belief that nonlaboratory data are "soft" assessment tools, and the fear of annoying patients with measurements [25]. The results observed may also be a consequence of poor medical record keeping. High-quality medical record keeping, containing detailed data on the patient and RA status, is essential to provide optimal patient care and assist in sharing information among healthcare professionals [26]. Further investigations into the characteristics of rheumatologists who perform RA measures frequently, compared with those who do not, are necessary to elucidate the exact reasons for these barriers.

In conclusion, this quality assurance review has shown an apparent lack of consistent RA activity measures being recorded in Australian rheumatology clinical practice. With the advent of bDMARDs and the demonstrated benefit of tight disease control [22], an accurate assessment of the disease process is necessary to treat patients with RA effectively. Index scores, such as DAS28, are a practical and easy to perform clinical-based method, and, unlike the ACR criteria, DAS28 allows individual responses to therapy to be assessed. Nevertheless, their use may need further guideline recommendations and appropriate education before they become embedded in routine rheumatology clinical practice.

\section{Conflict of Interests}

The authors received an honorarium from Roche Products Pty Limited for conducting this study.

\section{Acknowledgments}

This quality assurance review was sponsored by Roche Products Pty Limited (Australia). The authors would like to thank all rheumatologists (H. Bagga, P. Bird, A. Boers, J. O'Callaghan, P. Hanrahan, J. De Jager, J. Riordan, and A. Taylor), nurses, and other support staff involved in this review, and they are indebted to the patients who gave their consent to be involved in this review. The authors would like to thank Robert Liversage, PhD, from Publicis Life Brands, who provided medical writing support, funded by Roche Products Pty Limited (Australia).

\section{References}

[1] P. L. van Riel and H. Ralph Schumacher Jr., "How does one assess early rheumatoid arthritis in daily clinical practice?" Best Practice and Research, vol. 15, no. 1, pp. 67-76, 2001.

[2] M. A. Van Leeuwen, D. M. F. M. Van der Heijde, M. H. Van Rijswijk et al., "Interrelationship of outcome measures and process variables in early rheumatoid arthritis. A comparison of radiologic damage, physical disability, joint counts, and acute phase reactants," Journal of Rheumatology, vol. 21, no. 3, pp. 425-429, 1994.

[3] K. W. Drossaers-Bakker, M. De Buck, D. van Zeben, A. H. Zwinderman, F. C. Breedveld, and J. M. W. Hazes, "Long-term course and outcome of functional capacity in rheumatoid arthritis: the effect of disease activity and radiologic damage over time," Arthritis and Rheumatism, vol. 42, no. 9, pp. 18541860, 1999.

[4] J. J. Rasker and J. A. Cosh, "Cause and age at death in a prospective study of 100 patients with rheumatoid arthritis," Annals of the Rheumatic Diseases, vol. 40, no. 2, pp. 115-120, 1981.

[5] T. Pincus, Y. Yazici, and T. Sokka, "Quantitative measures of rheumatic diseases for clinical research versus standard clinical care: differences, advantages and limitations," Best Practice and Research, vol. 21, no. 4, pp. 601-628, 2007.

[6] D. T. Felson, J. J. Anderson, M. Boers et al., "The American College of Rheumatology preliminary core set of disease activity measures for rheumatoid arthritis clinical trials. The committee on outcome measures in rheumatoid arthritis clinical trials," Arthritis and Rheumatism, vol. 36, no. 6, pp. 729-740, 1993.

[7] D. T. Felson, J. J. Anderson, M. Boers et al., "American College of Rheumatology preliminary definition of improvement in rheumatoid arthritis," Arthritis and Rheumatism, vol. 38, no. 6, pp. 727-735, 1995.

[8] N. G. Ringold SaS, "Measures of disease activity in rheumatoid arthritis: a clinician's guide," Current Rheumatology Reviews, vol. 4, pp. 259-265, 2008.

[9] A. M. van Gestel, J. J. Anderson, P. L. van Riel et al., "ACR and EULAR improvement criteria have comparable validity in rheumatoid arthritis trials. American College of Rheumatology European League of Associations for Rheumatology," The Journal of Rheumatology, vol. 26, no. 3, pp. 705-711, 1999.

[10] M. L. L. Prevoo, M. A. Van't Hof, H. H. Kuper, M. A. van Leeuwen, L. B. A. van De Putte, and P. L. van Riel, "Modified disease activity scores that include twenty-eight-joint counts: development and validation in a prospective longitudinal study of patients with rheumatoid arthritis," Arthritis and Rheumatism, vol. 38, no. 1, pp. 44-48, 1995.

[11] D. Van Der Heijde, L. Klareskog, M. Boers et al., "Comparison of different definitions to classify remission and sustained remission: 1 Year TEMPO results," Annals of the Rheumatic Diseases, vol. 64, no. 11, pp. 1582-1587, 2005.

[12] M. Schoels, R. Knevel, D. Aletaha et al., "Evidence for treating rheumatoid arthritis to target: results of a systematic literature search," Annals of the Rheumatic Diseases, vol. 69, no. 4, pp. 638-643, 2010. 
[13] J. S. Smolen, D. Aletaha, J. W. J. Bijlsma et al., "Treating rheumatoid arthritis to target: recommendations of an international task force," Annals of the Rheumatic Diseases, vol. 69, no. 4, pp. 631-637, 2010.

[14] Australian Rheumatology Association, "Recommendations for the use of biological agents for the treatment of rheumatic diseases," 2009, http://www.rheumatology.org.au/otherpages/ biological-guidelines.asp.

[15] Medicare Australia, http://www.medicareaustralia.gov.au/provider/pbs/drugs2/rheumatoid.jsp.

[16] T. Neogi and D. T. Felson, "Composite versus individual measures of disease activity in rheumatoid arthritis," Journal of Rheumatology, vol. 35, no. 2, pp. 185-187, 2008.

[17] N. Bellamy, S. Kaloni, J. Pope, K. Coulter, and J. Campbell, "Quantitative rheumatology: a survey of outcome measurement procedures in routine rheumatology outpatient practice in Canada," Journal of Rheumatology, vol. 25, no. 5, pp. 852858, 1998.

[18] N. Bellamy, K. D. Muirden, P. M. Brooks, D. Barraclough, M. M. Tellus, and J. Campbell, "A survey of outcome measurement procedures in routine rheumatology outpatient practice in Australia," Journal of Rheumatology, vol. 26, no. 7, pp. 1593-1599, 1999.

[19] T. Pincus and O. G. Segurado, "Most visits of most patients with rheumatoid arthritis to most rheumatologists do not include a formal quantitative joint count," Annals of the Rheumatic Diseases, vol. 65, no. 6, pp. 820-822, 2006.

[20] M. Schoels, D. Aletaha, J. S. Smolen et al., "Follow-up standards and treatment targets in rheumatoid arthritis: results of a questionnaire at the EULAR 2008," Annals of the Rheumatic Diseases, vol. 69, no. 3, pp. 575-578, 2010.

[21] Y. P. M. Goekoop-Ruiterman, J. K. de Vries-Bouwstra, C. F. Allaart et al., "Clinical and radiographic outcomes of four different treatment strategies in patients with early rheumatoid arthritis (the best study): a randomized, controlled trial," Arthritis and Rheumatism, vol. 52, no. 11, pp. 3381-3390, 2005.

[22] C. Grigor, H. Capell, A. Stirling et al., "Effect of a treatment strategy of tight control for rheumatoid arthritis (the TICORA study): a single-blind randomised controlled trial," Lancet, vol. 364, no. 9430, pp. 263-269, 2004.

[23] S. M. M. Verstappen, J. W. G. Jacobs, M. J. van der Veen et al., "Intensive treatment with methotrexate in early rheumatoid arthritis: aiming for remission. Computer assisted management in early rheumatoid arthritis (CAMERA, an open-label strategy trial)," Annals of the Rheumatic Diseases, vol. 66, no. 11, pp. 1443-1449, 2007.

[24] T. Pham, L. Gossec, B. Fautrel et al., "Physical examination and laboratory tests in the management of patients with rheumatoid arthritis: development of recommendations for clinical practice based on published evidence and expert opinion," Joint Bone Spine, vol. 72, no. 3, pp. 222-228, 2005.

[25] J. Fransen, G. Stucki, and P. van Riel, "The merits of monitoring: should we follow all our rheumatoid arthritis patients in daily practice?" Rheumatology, vol. 41, no. 6, pp. 601-604, 2002.

[26] J. Fechtenbaum, F. Lecoq d'André, H. Nataf et al., "Practice patterns in outpatient rheumatology: a pilot evaluation of medical file content," Joint Bone Spine, vol. 74, no. 2, pp. 171174, 2007. 


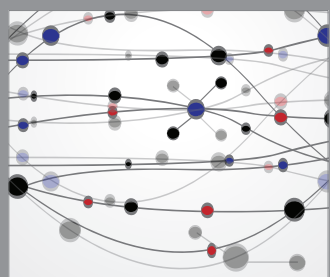

The Scientific World Journal
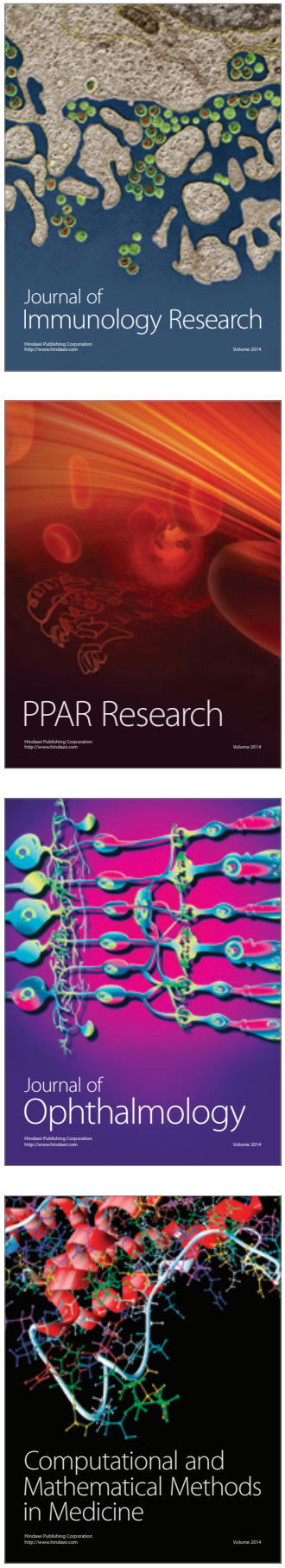

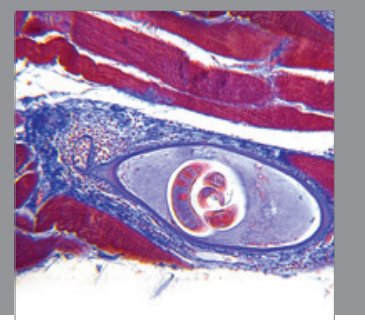

Gastroenterology

Research and Practice
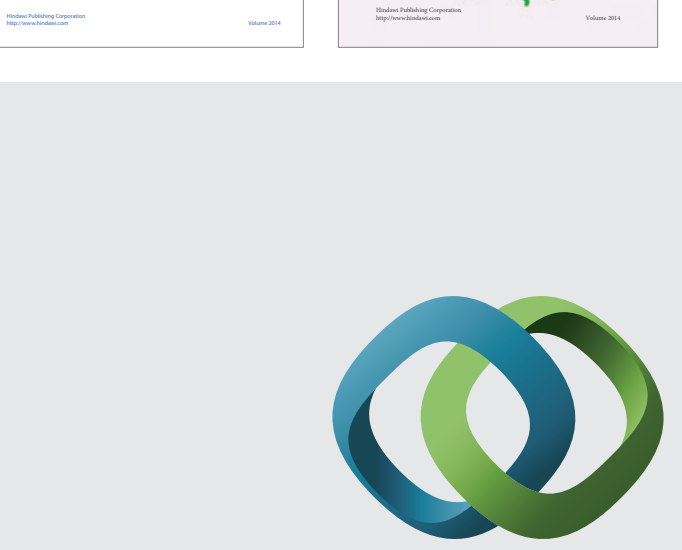

\section{Hindawi}

Submit your manuscripts at

http://www.hindawi.com
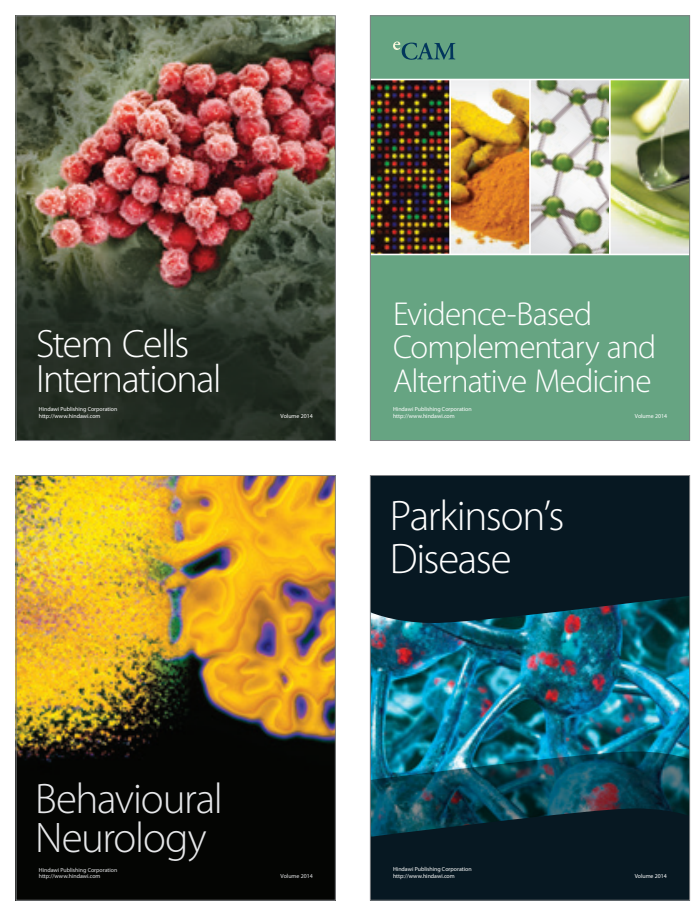

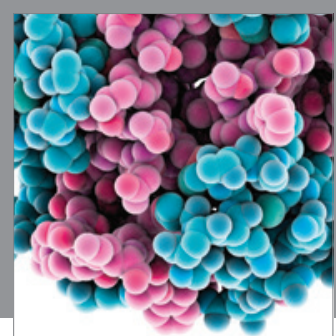

Journal of
Diabetes Research

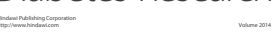

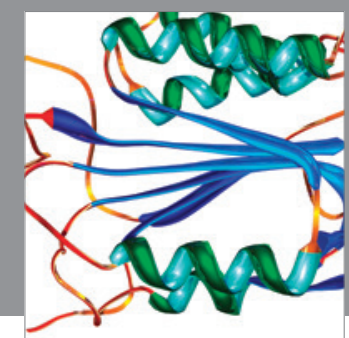

Disease Markers
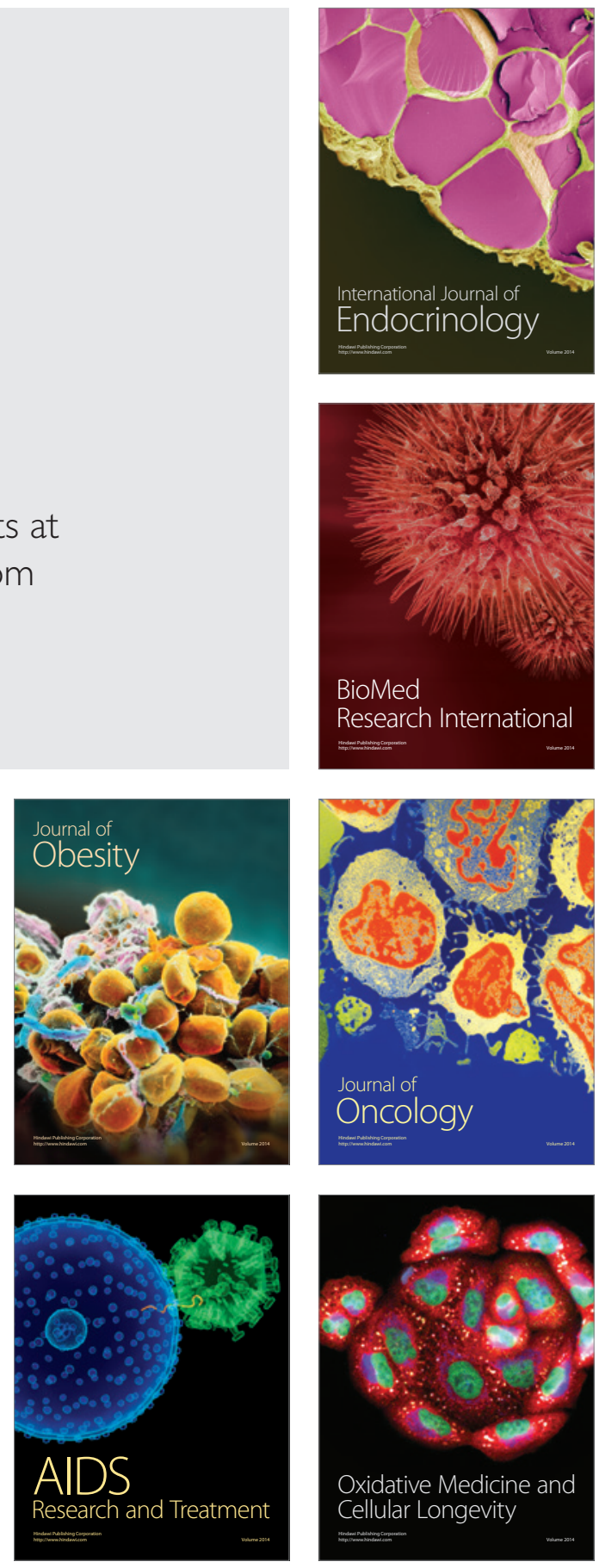